\title{
Non-Cooperative Feedback Control Game for Secondary Transmitter in Cognitive Radio Network
}

\author{
Jungho Myung, Student Member, IEEE, Yan Chen, Member, IEEE, K. J. Ray Liu, Fellow, IEEE, and \\ Joonhyuk Kang, Member, IEEE
}

\begin{abstract}
In this letter, we propose a non-cooperative feedback control game for secondary transmitter, where we assume that secondary users are rational and thus selfish, i.e., they will choose the feedback rate to maximize their own utility functions. The utility function of each user is defined as the downlink data-rate minus a linear price function of the channel state information (CSI) feedback rate. The existence of the Nash-equilibrium of the proposed game is analyzed. Also, in order to satisfy the primary user's interference threshold, power control algorithms are proposed. Simulation results show that the sum-rate of the proposed game is better than that of the equally distributed feedback-size scheme.
\end{abstract}

Index Terms-Cognitive radio network, game thoery, limited feedback, non-cooperative control game.

\section{INTRODUCTION}

$\mathbf{N}$ OWADAYS, due to the emergence of high-speed communication, the demand for spectral resources has increased dramatically. On the other hand, the existing spectral utilization can be as low as $15 \%$ according to the report of the Federal Communication Commission [1]. Therefore, it is very important to develop efficient methods to manage and share the spectral resources. This motivates the development of Cognitive Radio (CR) [2]-[4], especially in the underlay CR network, where the secondary users (SUs) can always access the spectrum no matter whether the spectrum is used or not used by the primary users (PUs). However, there is a restriction that the interference received at the PUs due to the secondary transmission should be less than a pre-determined threshold. In the literature, there are many related works such as capacity analysis and secondary transmitter design [5]-[8].

With multiple antennas as the transmitter, multiple users can be simultaneously served through multiplexing [9]-[12]. To achieve this, CSI at the transmitter is necessary for designing precoding matrix to control inter-user-interference (IUI). Generally, CSI can be obtained by feedback from receivers

Manuscript received March 07, 2013; accepted March 26, 2013. Date of publication April 12, 2013; date of current version April 18, 2013. This work was supported by the MKE (Ministry of Knowledge Economy), Korea, under the R\&D program supervised by the KEIT (Korea Evaluation Institute of Industrial Technology) (KEIT-2013-(30-10041818)). The associate editor coordinating the review of this manuscript and approving it for publication was Prof. Min Dong.

J. Myung and J. Kang are with the Department of Electrical Engineering, Korea Advanced Institute of Science and Technology, Daejeon 350-732, Korea (e-mail: jhmyung81@kaist.ac.kr; jhkang@ee.kaist.ac.kr).

Y. Chen and K. J. R. Liu are with the Department of Electrical and Computer Engineering, University of Maryland, College Park, MD 20742 USA (e-mail: yan@umd.edu; kjrliu@umd.edu).

Color versions of one or more of the figures in this paper are available online at http://ieeexplore.ieee.org.

Digital Object Identifier 10.1109/LSP.2013.2257755 in frequency division duplexing. However, since the feedback-rate is generally limited, perfect CSI cannot be obtained at the transmitter. Therefore, it is hard to control the IUI perfectly in practice. In the literature, there are many related works such as capacity analysis of secondary links under imperfect CSI [7], [8], [13]. Since these works treated each user independently and focused on the multi-user CSI feedback problem from information theory point of view, each SU sends its own CSI to transmitter using equal-sized and fixed feedback bits. However, since there are competitions in effective CSI feedback-rates among SUs under sum feedback-rate constraint, equal-sized CSI feedback model is obviously inefficient. Hence, it will be desirable to sort out the competition by finding an equilibrium in the multiple SUs feedback scenario. To analyze the competition and cooperation behavior in CR network, game theory has been widely used [4], [14], [15]. However, most of existing works assume the CSI is perfect and known while in practice the CSI is imperfect due to limited feedback. The most related work is [16] where the authors use game theory to analyze channel feedback for multiple input multiple output (MIMO) system. Nevertheless, it is different from our work since it neither focuses on CR network nor considers sum feedback-rate constraint.

In this letter, we propose a non-cooperative feedback control game for secondary transmitter design under sum feedback-rate constraint. In the proposed game, the utility function of a SU is determined by the downlink data-rate and CSI feedback size. Since SUs are rational and thus selfish, they will determine the feedback-rate by maximizing their own utility functions under the sum feedback-rate constraint. By analyzing the proposed game, we discuss the existence of the Nash equilibrium. Moreover, we propose power control algorithms to guarantee the interference threshold at the PUs. Simulation results show that the sum-rate of the proposed game is better than that of the equally distributed feedback size scheme. Furthermore, with the proposed power control algorithms, the interference restriction at the PUs is guaranteed under imperfect PUs' channel condition.

Throughout the letter, we use the following notation. Let $\mathbf{x}^{T}$, $\mathbf{x}^{-1}$, and $\|\mathbf{x}\|$ denote the transpose, pseudo-inverse and the Euclidean norm of vector $\mathbf{x}$, respectively.

\section{PRELIMINARIES}

We consider an underlay CR network with $N_{p}$ PUs, $N_{s}$ SUs, and one secondary base station (SB) with $N_{t}\left(>N_{p}\right)$ antennas. Similar to [17], we assume that each SU can perfectly estimate the downlink CSI and send it back to SB using limited feedback under sum feedback rate constraint. Moreover, we assume that there exists cooperative channel feedback between primary user 
(PU) and secondary base station (SB) [13]. Through the cooperative feedback, the SB can transmit data to SUs and control the interferences to the PUs.

For the $k$-th $\mathrm{SU}$, the transmitted signal, $x_{k}$, is precoded by precoding vector $\mathbf{f}_{k}$ from $N_{t}$ antennas at the SB. Therefore, the received signal $y_{k}$ at the $k$-th SU can be expressed as

$$
y_{k}=\mathbf{h}_{s_{k} b}^{T} \mathbf{f}_{k} x_{k}+\mathbf{h}_{s_{k} b}^{T} \sum_{i=1, i \neq k}^{K} \mathbf{f}_{i} x_{i}+n_{k},
$$

where $\mathbf{h}_{s_{k} b}, n_{k}$, and $K$ denote the channel vector from the SB to the $k$-th SU, the additive white Gaussian noise (AWGN) $C N\left(0, N_{0}\right)$, and the number of selected SUs for transmission, resepctively. The channel is assumed to be Rayleigh flat fading, where the elements of each user's channel vector are independent complex Gaussian random variables with zero mean and unit variance.

Assuming equal power allocation and zero-forcing (ZF) for precoder design, the sum-rate of the $k$-th $\mathrm{SU}$ is given by

$$
C_{k}=\log _{2}\left(1+\frac{\left\|\mathbf{h}_{s_{k} b}^{T} \mathbf{f}_{k}\right\|^{2} \frac{P_{s}}{K}}{\left\|\mathbf{h}_{s_{k} b}^{T} \sum_{i=1, i \neq k}^{K} \mathbf{f}_{i}\right\|^{2} \frac{P_{s}}{K}+N_{0}}\right),
$$

where $P_{s}$ denotes the secondary transmit power.

Due to the secondary transmission, there is an interference received at the PUs. The interference at the $p$-th $\mathrm{PU}, I_{p}$, can be written as

$$
I_{p}=\left\|\sum_{i=1}^{K} \mathbf{h}_{p_{p} b}^{T} \mathbf{f}_{i} x_{i}\right\|^{2},
$$

where $\mathbf{h}_{p_{p} b}$, denotes the channel vector from the SB to the $p$-th PU. Notice that $I_{p}$ must be smaller than a pre-defined threshold $I_{t h}$ to guarantee the quality-of-service of PUs.

Also, we assume the quantized CSI can be modeled as [16]

$$
\overline{\mathbf{h}}_{s_{k} b}=\sqrt{1-2^{-r_{k}}} \mathbf{h}_{s_{k} b}+\sqrt{2^{-r_{k}}} \overline{\mathbf{n}}_{f},
$$

where $\overline{\mathbf{n}}_{f}$ is i.i.d. Gaussian vector with $C N(0,1)$.

\section{Secondary Transmit Power Control}

Considering equal power allocation and ZF precoding, the maximum interference at the $p$-th primary user can be expressed by

$$
I_{p}=\max \left(\left\|\mathbf{h}_{p_{p} b}^{T}\right\|^{2} \cdot 2^{-r_{p}} \cdot P_{s}\right), \forall p=1,2, \ldots, N_{p} .
$$

where $r_{p}$ is the feedback rate of the $p$-th PU transmitted from user to SB in order to be satisfied with the interference constraint $I_{t h}\left(\geq I_{p}\right)$. Then, the optimal transmit power $P_{s}^{*}$ can be obtained as

$$
P_{s}^{*}=\min \left(P_{s}, P_{s, \max }\right)
$$

where

$$
P_{s}=\frac{I_{t h}}{\max \left(\left\|\mathbf{h}_{p_{p} b}^{T}\right\|^{2} \cdot 2^{-r_{p}}\right)}, \forall p=1,2, \ldots, N_{p} .
$$

and $P_{s, \text { max }}$ is the maximum secondary transmit power. If $r_{p} \rightarrow$ $\infty, P_{s}^{*}$ becomes $P_{s, \max }$. However, since $r_{p}$ is finite in practice, the transmit power is controlled.

\section{Non-Cooperative Feedback Control Game}

\section{A. User's Utility Function}

To achieve the rate in (2), the $k$-th SU needs to send the quantized CSI back to SB. In other words, the SU needs to pay a certain cost of sending the feedback to achieve the sum-rate. Therefore, the utility function of $k$-th secondary user can be defined as

$$
U_{k}\left(r_{k}\right)=C_{k}\left(r_{k}\right)-\alpha r_{k},
$$

where $C_{k}\left(r_{k}\right)$ is the rate the $k$-th $\mathrm{SU}$ can achieve when using feedback rate $r_{k}, \alpha$ is a parameter controlling the balance between the gain, $C_{k}\left(r_{k}\right)$, and the cost, $r_{k}$. Here we assume a linear cost function.

Since the SB uses the ZF precoder, SU can estimate the interference $I_{k}$ due to channel feedback error and the beamforming vectors of other users as

$$
I_{k}=\left\|\mathbf{h}_{s_{k} b}^{T} \sum_{i=1, i \neq k}^{K} \mathbf{f}_{i}\right\|^{2}=(K-1)\left\|\mathbf{h}_{s_{k} b}^{T}\right\|^{2} \sin ^{2}\left(\theta_{k, \max }\right),
$$

where $\theta_{k, \max }$ is the maximum angle error using the feedbackrate $r_{k}$. With $\theta_{k, \text { max }}$, the user can also estimate the desired signal power $\left\|\mathbf{h}_{s_{k} b}^{T} \mathbf{f}_{k}\right\|^{2}$. Therefore, the $C_{k}\left(r_{k}\right)$ can be re-written as follows

$$
C_{k}\left(r_{k}\right)=\log _{2}\left(1+\frac{\cos ^{2}\left(\theta_{o p t}+\theta_{k, \max }\right)}{(K-1) \sin ^{2}\left(\theta_{k, \text { max }}\right)+\frac{K N_{0}}{P_{s}^{*}\left\|\mathbf{h}_{s_{k} b}^{T}\right\|^{2}}}\right),
$$

where

$$
\theta_{o p t}=\cos ^{-1} \frac{\left\|\mathbf{h}_{s_{k} b}^{T} \mathbf{f}_{k}\right\|}{\left\|\mathbf{h}_{s_{k} b}^{T}\right\|}-\theta_{k, \max }, \sin ^{2}\left(\theta_{k, \text { max }}\right)=2^{-r_{k}} .
$$

As $r_{k}$ goes to infinite, $\theta_{k, \max }$ goes to zero and $\mathbf{f}_{k}$ becomes the optimal precoding vector, which leads to the maximal rate $C_{k}$. Note that interference in $C_{k}\left(r_{k}\right)$ is independent from other SUs' CSI. Therefore, we can calculate the $\theta_{o p t}$ easily.

\section{B. Game Formulation}

Since SUs are naturally selfish, they try to maximize their own utility function in (8) under the sum feedback-rate constraint. We assume that the $k$-th SU has a minimum feedbackrate constraint $r_{k}^{\min }$ to guarantee a minimal performance and a maximum feedback-rate constraint $r_{k}^{\max }$ to achieve the maximum performance. Obviously, if the sum feedback-rate constraint is able to satisfy all SUs with the maximum feedback-rate 


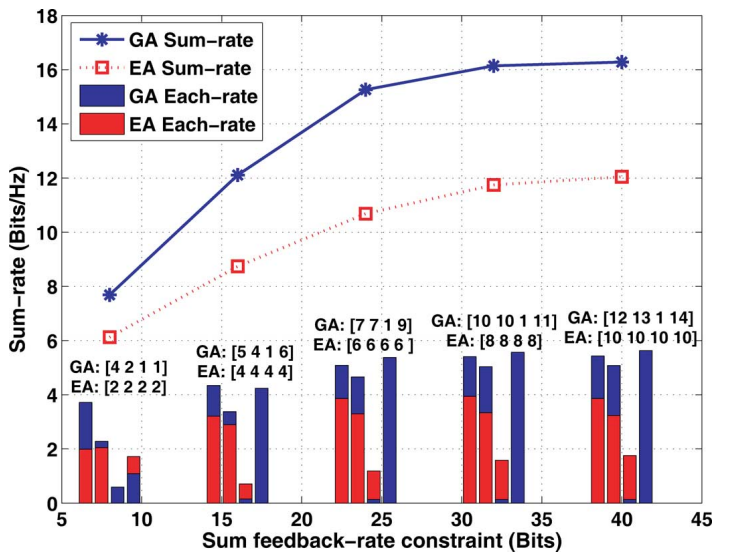

Fig. 1. Sum-rate vs. Sum feedback-rate constraint where $N_{t}=K=4, N_{p}=$ $0, P_{s}=20 \mathrm{~dB}$.

$r_{k}^{\max }$, the feedback-rate allocation problem is trivial since the SB just allocates $r_{k}^{\max }$ to each SU. The allocation problem becomes more interesting when the sum feedback-rate constraint cannot provide all SUs with the maximum feedback-rate constraint $r_{k}^{\max }$. From the SUs' point of view, they try to maximize their utility function subject to the constraint that the sum of the users' feedback-rate does not exceed the sum feedback-rate $R_{\text {total }}$. Therefore, the game can be formulated as

$$
\begin{aligned}
\max _{r_{k}} \quad U_{k}\left(r_{k}\right) & =C_{k}\left(r_{k}\right)-\alpha r_{k} \\
\text { s.t. } \quad r_{k}^{\min } & \leq r_{k} \leq r_{k}^{\max }, \forall k=1,2, \ldots, K \\
\sum_{k=1}^{K} r_{k} & =R_{\text {total }}
\end{aligned}
$$

where $R_{\text {total }}$ denotes the available sum feedback-rate.

\section{Analysis of the Game}

According to (8) and (10), we can see that the utility function $U_{k}\left(r_{k}\right)$ is a concave function in terms of $r_{k}$. Therefore, the $k$-th SU achieves its maximal utility at $r_{k}^{*}$, where $r_{k}^{*}$ is defined as [18]

$$
\begin{aligned}
r_{k}^{*} & =\max \left[r_{k}^{\min }, \min \left(r_{k}^{t e m p}, r_{k}^{\max }\right)\right] \\
\forall k & =1,2, \ldots, K
\end{aligned}
$$

with $r_{k}^{\text {temp }}$ satisfying

$$
\left.\frac{\partial U_{k}\left(r_{k}\right)}{\partial r_{k}}\right|_{r_{k}=r_{k}^{t e m p}}=C_{k}^{\prime}\left(r_{k}^{t e m p}\right)-\alpha=0 .
$$

Since the optimal $r_{k}^{*}$ is determined by the parameter $\alpha$, the game has different equilibria with different physical meanings for different choices of $\alpha$. Especially, we discuss the Nash equilibrium in three different cases: $\alpha>\alpha^{\max }, \alpha^{\min } \leq \alpha \leq \alpha^{\max }$, and $0 \leq \alpha<\alpha^{\min }$, where $\alpha^{\min }$ and $\alpha^{\max }$ are the constant satisfying

$$
\begin{array}{r}
\sum_{k=1}^{K} \max \left[r_{k}^{\min }, \min \left(\left.r_{k}^{t e m p}\right|_{\alpha^{\min } \leq \alpha \leq \alpha^{\max }}, r_{k}^{\max }\right)\right] \\
=R_{\text {total }} .
\end{array}
$$

1) Non-Nash Equilibrium Allocation $\left(0 \leq \alpha<\alpha^{\text {min }}\right)$ : In this case, since the $\sum_{k=1}^{K} r_{k}^{*}>R_{\text {total }}$, the game cannot achieve the desired Nash equilibrium.
2) Non-Efficient Rate Allocation $\left(\alpha>\alpha^{\max }\right)$ : In this case, the game has a unique Nash equilibrium. However, since $\alpha>$ $\alpha^{\text {max }}$, from (19), the sum of the SUs' feedback rate is smaller than $R_{\text {total }}, \sum_{k=1}^{K} r_{k}^{*}<R_{\text {total }}$, i.e., the available sum feedback rate is not fully utilized. Therefore, this allocation scheme is not efficient.

3) Efficient Rate Allocation $\left(\alpha^{\min } \leq \alpha \leq \alpha^{\max }\right)$ : If $\alpha^{\min } \leq$ $\alpha \leq \alpha^{\max }$, the game has a unique Nash equilibrium which satisfies $\sum_{k=1}^{K} r_{k}^{*}=R_{\text {total }}$, i.e., the available sum feedback rate constraint is fully utilized. Therefore, this allocation scheme is efficient. To achieve the efficient Nash equilibrium, we propose a simple algorithm to find the proper $\alpha$ as shown in Algorithm 1 .

\section{Algorithm 1: Algorithm for Non-Cooperative Feedback} Control Game

- Base station:

1. Set $\alpha=0$ and announce $\alpha$ and $K$ to all SUs

- Each user:

2. Find $r_{k}^{*}=\max \left[r_{k}^{\min }, \min \left(r_{k}^{t e m p}, r_{k}^{\max }\right)\right]$ and report $r_{k}^{*}$ to $\mathrm{SB}$

- Base station:

3. If $\sum_{k=1}^{K} r_{k}^{*}>R_{\text {total }}$

- Increase $\alpha:=\alpha+\Delta \alpha$ and announce to all SUs

- Go to step 2

Else $\sum_{k=1}^{K} r_{k}^{*}=R_{\text {total }}$

- Stop and announce $\alpha^{\text {opt }}=\alpha$.

\section{Simulation Results}

We compare the proposed game theoretic feedback-rate allocation scheme (GA) with equal feedback-rate allocation scheme (EA) and the optimal feedback-rate allocation scheme (OA) that maximizes the sum-rate. Note that OA does not consider the selfish nature of SUs and thus there is no concept of equilibrium, i.e., SUs may not be willing to follow the strategy obtained by $\mathrm{OA}$.

In Fig. 1, we show the sum-rate performance under instantaneous channel condition. We can see when the sum feedbackrate increases, the feedback CSI becomes more accurate, which leads to the increase of the sum-rate of all SUs. By dynamically allocating the feedback size, the the proposed GA achieves much better performance compared with EA which equally allocates the feedback size.

In Fig. 2, we evaluate the performance of sum-rate under different SNR by fixing sum feedback-rate $R_{\text {total }}$ to be 12 . We can see that the sum-rates are saturated in high SNR region due to the interference. However, with the efficient feedback-rate allocation, the saturation point of the proposed GA is much higher than that of EA, i.e., the achievable sum-rate of the proposed GA is larger than that of EA.

In Fig. 3, the average sum-rate performance versus sum feedback-rate is evaluated at $P_{s}=20 \mathrm{~dB}$ for different approaches. We can see that both GA and OA achieve much better performance compared with EA. Note that the OA requires all SUs feedback the SINR to the SB as well as a full search algorithm for the power allocation, which can be very costly. With the proposed GA method, we can achieve similar performance with much lower cost. Moreover, OA does not take into account the 


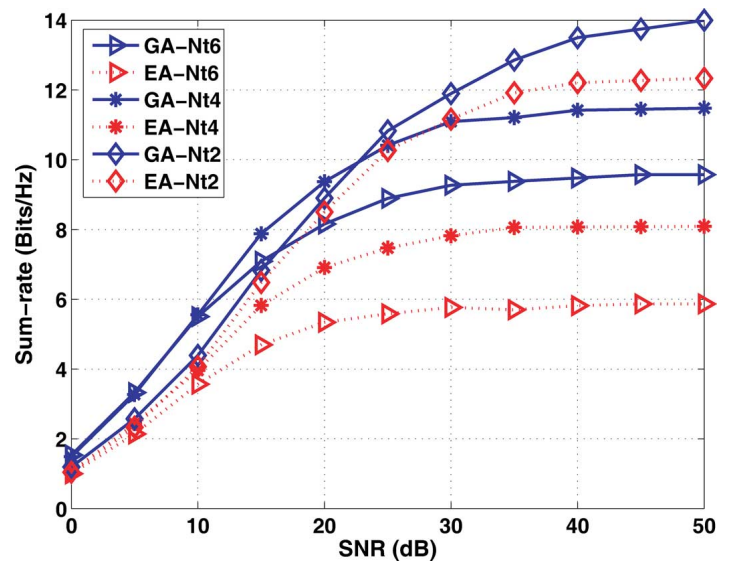

Fig. 2. Sum-rate vs. SNR corresponding feedback-rate allocation type, where $N_{t}=K=\{2,4,6\}$ and $R_{\text {total }}=12$.

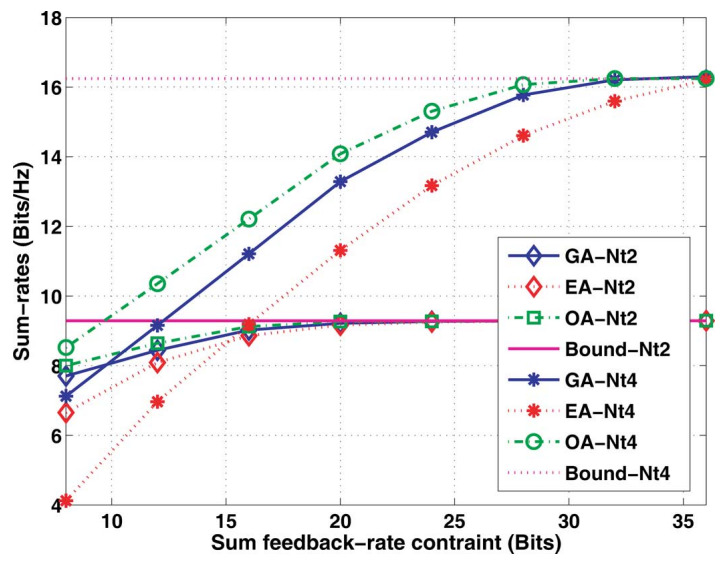

Fig. 3. Sum-rate vs. Sum feedback-rate contraint corresponding feedback bit allocation type, where $N_{t}=K=\{2,4\}$ and $P_{s}=20 \mathrm{~dB}$.

selfish nature of SUs. In such a case, selfish SUs will not follow the strategy obtained by OA since they may obtain higher payoffs by deviating.

Fig. 4 shows the performance of the proposed scheme with different feedback bits from PUs. When the feedback bits are large, e.g., 10, the transmit power restriction of SUs is loose according to (7). In such a case, there is little performance degradation compared to the perfect PUs' CSI case. However, if feedback bits are small, due to the secondary transmit power restriction, there is large performance degradation. Therefore, choosing a proper feedback bits from PUs is very important and will be one of our future directions.

\section{CONCLUSIONS}

In this letter, the secondary transmitter design based on noncooperative feedback control game is proposed. Through the proposed game, SUs can efficiently determine their own optimal feedback-rate to maximize their own utilities. Simulation results show the advantage of the proposed scheme compared with the equal feedback size scheme. Moreover, we also found that the feedback bits from PUs play an important role in our scheme. Therefore, in the future, we would like to extend our current scheme with fixed feedback bits from PUs to a dynamic scheme where PUs can determine their own feedback bits to maximize their own utilities.

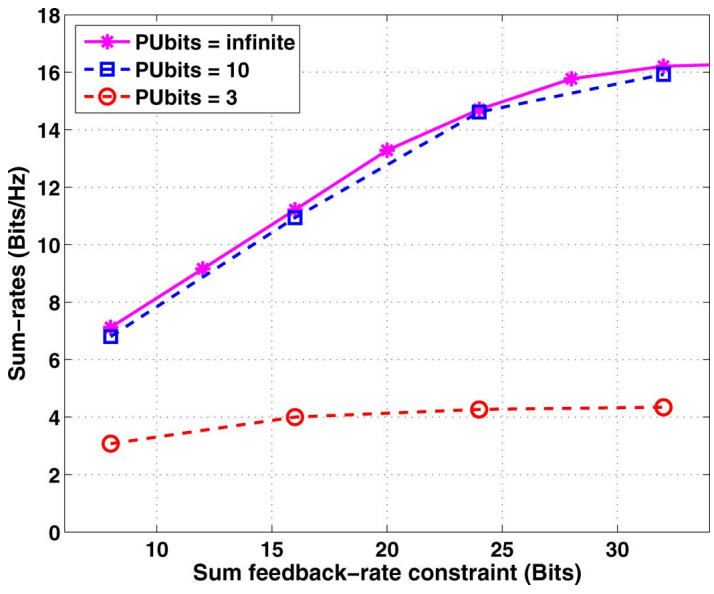

Fig. 4. Sum-rate vs. Sum feedback-rate contraint with different feedback bits from PUs, where $N_{p}=1, N_{t}=K+N_{p}=5, P_{s}=20 \mathrm{~dB}$.

\section{REFERENCES}

[1] Facilitating Opportunities for Flexible, Efficient, and Reliable Spectrum Use Employing Cognitive Radio Technologies, Notice of Proposed Rule Making and Order FCC, 2003, FCC 03-322.

[2] S. Haykin, "Cognitive radio: brain-empowered wireless communications," IEEE J. Sel. Areas Commun., vol. 23, no. 2, pp. 201-220, Feb. 2005.

[3] B. Wang and K. J. R. Liu, "Advances in cognitive radio networks: A survey,” IEEE J. Sel. Topics Signal Process., vol. 5, no. 1, pp. 5-23, Feb. 2011.

[4] K. J. R. Liu and B. Wang, Cognitive Radio Networking and Security: A Game Theoretical View. Cambridge, U.K.: Cambridge Univ. Press, 2010.

[5] M. Gastpar, "On capacity under receive and spatial spectrum-sharing constraints," IEEE Trans. Inf. Theory, vol. 53, no. 2, pp. 471-487, Feb. 2007.

[6] N. Devroye, P. Mitran, and V. Tarokh, "Achievable rates in cognitive radio channels," IEEE Trans. Inf. Theory, vol. 52, no. 5, pp. 1813-1827, May 2006.

[7] R. Zhang and Y.C. Liang, "Exploiting multi-antennas for opportunistic spectrum sharing in cognitive radio networks," IEEE J. Sel. Topics Signal Process., vol. 2, no. 1, pp. 88-192, Feb. 2008.

[8] H. Suraweera, P. Smith, and M. Shafi, "Capacity limits and performance analysis of cognitive radio with imperfect channel knowledge," IEEE Trans. Veh. Technol., vol. 59, no. 4, pp. 1811-1822, May 2010.

[9] N. Jindal, "MIMO broadcast channels with finite-rate feedback," IEEE Trans. Inf. Theory, vol. 52, no. 11, pp. 5045-5060, Sep. 2006.

[10] N. Ravindran and N. Jindal, "Limited feedback-based block diagonalization for the MIMO broadcast channel," IEEE J. Sel. Areas Commun., vol. 26 , no. 8, pp. 1473-1482, Oct. 2008.

[11] D. J. Love and R. W. Heath, "Limited feedback unitary precoding for spatial multiplexing systems," IEEE Trans. Inf. Theory, vol. 51, no. 8, pp. 2967-2976, Aug. 2005.

[12] R. W. Heath and D. J. Love, "Multimode antenna selection for spatial multiplexing systems with linear receivers," IEEE Trans. Signal Process., vol. 53, no. 8, pp. 3042-3056, Aug. 2005.

[13] K. Huang and R. Zhang, "Cooperative feedback for multiantenna cognitive radio networks," IEEE Trans. Signal Process., vol. 59, no. 2, pp. 747-758, Feb. 2011.

[14] B. Wang, Y. Wu, and K. J. R. Liu, "Game theory for cognitive radio networks: An overview," Comput. Netw., vol. 54, no. 14, pp. 2537-2561, Oct. 2010.

[15] Q. Ni and C. Zarakovitis, "Nash bargaining game theoretic scheduling for joint channel and power allocation in cognitive radio systems," IEEE J. Sel. Areas Commun., vol. 30, no. 1, pp. 70-81, Jan. 2012.

[16] L. Song, Z. Han, Z. Zhang, and B. Jiao, "Non-cooperative feedbackrate control game for channel state information in wireless networks," IEEE J. Sel. Areas Commun., vol. 30, no. 1, pp. 188-197, Jan. 2012.

[17] K.-B. Huang, R. W. Heath, Jr., and J. G. Andrews, "Space division multiple access with a sum feedback rate constraint," IEEE Trans. Signal Process., vol. 55, no. 7, pp. 3879-3891, Jul. 2007.

[18] Y. Chen, B. Wang, and K. J. R. Liu, "Multi-user rate allocation games for multimedia communications," IEEE Trans. Multimedia, vol. 11, no. 6, pp. 1170-1181, Oct. 2009. 